\title{
Temperature Dependence of Exciton and Trion States in CdTe Quantum Well at High Magnetic Fields
}

\author{
D. Andronikovi, ${ }^{a}$, V. Kochereshioo ${ }^{a}$, A. Platonov $^{a}$, \\ S. Crooker $^{b}$, T. Barrick ${ }^{b}$ And G. KArCzewski ${ }^{c}$ \\ ${ }^{a}$ A.F. Ioffe Physical-Technical Institute, RAS \\ Politechnicheskaya 26, 194021, St-Petersburg, Russia \\ ${ }^{b}$ National High Magnetic Field Laboratory \\ MS E536 Los Alamos, New Mexico 87545, USA \\ ${ }^{c}$ Institute of Physics, Polish Academy of Sciences \\ al. Lotników 32/46, 02-668 Warsaw, Poland
}

\begin{abstract}
Temperature dependent photoluminescence and reflectivity spectra of excitons, singlet and triplet states of trions were studied in CdTe-based quantum wells at high magnetic fields. Interesting features of the exciton and trion spectral lines with altering temperatures are explained by analyzing the thermal population and the kinetics of the exciton-electron-trion energetic system.
\end{abstract}

PACS numbers: 71.35.Ji, 73.21.Fg, 78.55.Et, 78.67.De

\section{Introduction}

The problem of multiple-electron complexes is one of the most fundamental problems of physics. One of the examples of such complex is a negatively charged exciton (trion), which consists of three particles: two electrons and a hole. Trion states have been under investigation for more than a decade and are now well-studied objects. Still some problems remain unresolved. There are arguments why are triplet trion states, which are known to be dark, observed. To get a better understanding of this one should consider the formation mechanisms of

${ }^{*}$ corresponding author 
singlet and triplet trions. These mechanisms are strongly influenced by magnetic fields and changing temperature.

In the present work singlet and triplet trion states were studied in CdTe-based quantum well (QW) structures at different temperatures. Interesting peculiarities of the behavior of the photoluminescence (PL) lines of the exciton, singlet, dark and bright triplet trion states have been observed. In order to explain these peculiarities we have performed a model calculation of the exciton-trion energetic system taking into account the thermal population of all the energy levels and the trion formation mechanisms.

\section{Experiment}

We studied $\mathrm{CdTe} / \mathrm{Cd}_{0.7} \mathrm{Mg}_{0.3} \mathrm{Te}$ heterostructures with a single $100 \AA \mathrm{QW}$ MBE grown on the (100) GaAs substrate.

A iodine-doped $n$-type $\delta$-layer is located $100 \AA$ from the well. At low temperatures electrons from the $\delta$-layer are collected in the quantum well forming a quasi-2D electron gas (2DEG). We studied a set of such heterostructures with electron concentrations in the QW varied within the range from $10^{10} \mathrm{~cm}^{-2}$ to $10^{12} \mathrm{~cm}^{-2}$. The samples were not photosensitive, i.e. the electron concentration did not depend on the power of the additional illumination.

Polarized PL and reflectivity from these samples were measured with magnetic fields applied in the Faraday configuration. A capacitor-driven $50 \mathrm{~T}$ mid-pulse magnet (400 ms pulse duration) was used to yield high magnetic fields. A complete set of field-dependent PL spectra excited by a semiconductor diode laser with $\lambda=532 \mathrm{~nm}$ at $1.6 \mathrm{~K}, 4.2 \mathrm{~K}$, and $15 \mathrm{~K}$ temperature was collected during each magnet pulse. Optical fibers were used for optical illumination of the sample, and the emitted light was detected in both circular polarizations $\sigma^{+}$and $\sigma^{-}$allowing identification of the spin components of excitons and trions. A similar setup was used for reflectivity measurements with a halogen lamp applied for illumination of the sample.

\section{Results and discussion}

\subsection{Luminescence}

A set of luminescence spectra registered from the sample with electron concentration $n_{\mathrm{e}}=3 \times 10^{10} \mathrm{~cm}^{-2}$ in the range of magnetic fields from 0 to $45 \mathrm{~T}$ in left circular polarization $\sigma^{-}$is shown in Fig. 1. The PL spectra in $\sigma^{+}$polarization are not shown because the signals are too weak.

At the temperature of $1.6 \mathrm{~K}$ in low magnetic field (close to zero) a bright PL singlet trion line $T_{\mathrm{S}}$ is observed in $\sigma^{-}$circular polarization. In low magnetic field the neutral exciton line $X$ is visible only as a small shoulder. With the growth of the magnetic field the intensity of the luminescence line of the singlet trion state drops as the intensity of the exciton line rises. Such behavior is caused by the 


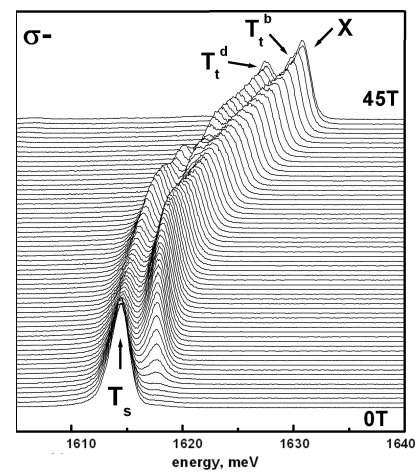

Fig. 1. Set of photoluminescence spectra taken in the magnetic fields from 0 to $45 \mathrm{~T}$ from the sample with $n_{\mathrm{e}}=3 \times 10^{10} \mathrm{~cm}^{-2}$ in circular polarization. $X-$ the exciton line, $T_{\mathrm{s}}$ - the singlet trion line, $T_{\mathrm{t}}^{\mathrm{d}}-$ the dark triplet trion line, $T_{\mathrm{t}}^{\mathrm{b}}-$ the bright triplet trion line.

suppression of the singlet state formation mechanism by the magnetic field. At higher temperatures $(4.2$ and $15 \mathrm{~K}$ ) the intensity redistributes towards the trion $\mathrm{PL}$ line $T_{\mathrm{s}}$. At $5 \mathrm{~T}$ magnetic field at $1.6 \mathrm{~K}$ the intensity of the trion line is about the same as the intensity of the exciton line, at $4.2 \mathrm{~K}$ the trion line intensity is about 4 times that of the exciton line and at $15 \mathrm{~K}$ this ratio is equal to $10 \div 11$ (see Fig. 2). This means that at higher temperatures the suppression of the singlet trion state formation mechanism by the magnetic field weakens. At temperatures as low as $1.6 \mathrm{~K}$ only the lower Zeeman sublevels of the excitons and electrons are populated in the magnetic field. But the singlet trion state is formed from the higher exciton and electron sublevels [1]. At higher temperatures these sublevels start to get populated which leads to the increase in the trion PL and subsequently to decrease in the PL of the neutral exciton (the integral intensity does not change).

At the magnetic field of $21 \mathrm{~T}$ a new line $T_{\mathrm{t}}^{\mathrm{d}}$ appears in the spectra in $\sigma^{-}$ circular polarization (Fig. 1). We attribute this line to the dark triplet state of the trion. With the growth of the magnetic field its intensity rises. As the temperature rises this new line appears in the spectra at higher magnetic field. As seen in Fig. 3, at $4.2 \mathrm{~K}$ it becomes evident only at $35 \mathrm{~T}$ magnetic field and at $15 \mathrm{~K}$ is not seen in the spectra at all. This is due to the peculiarities of the triplet trion state formation. Contrary to the singlet trion, the triplet trion is formed from the lower exciton and electron Zeeman sublevels [2]. Hence when the temperature increases the lower electron and exciton Zeeman sublevels become less populated to the favor of the higher ones. The triplet trion state becomes the less populated the higher the temperature is, which leads to the decrease in its PL at the given magnetic field.

It was interesting to examine the behavior of the intensity of the singlet trion PL line at the range of the magnetic field between 23 and $27 \mathrm{~T}$ in $\sigma^{-}$circular 
Fig. 2

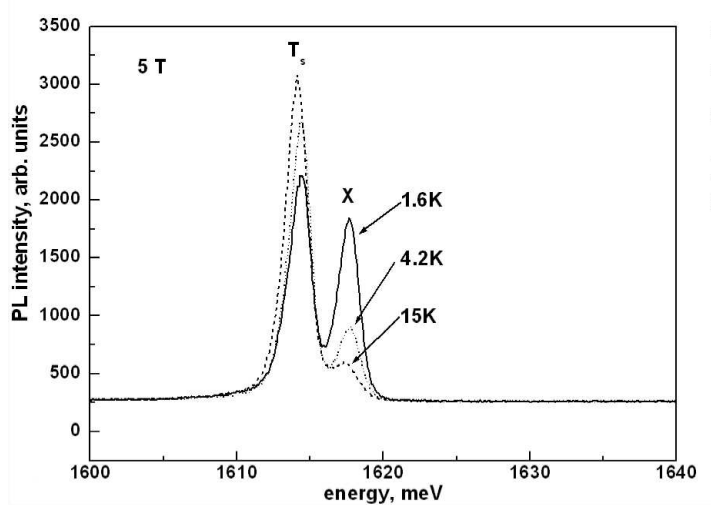

Fig. $33000-28 \mathrm{~T} \quad T^{d}:-T^{\mathrm{T}=1.6 \mathrm{~K}}$

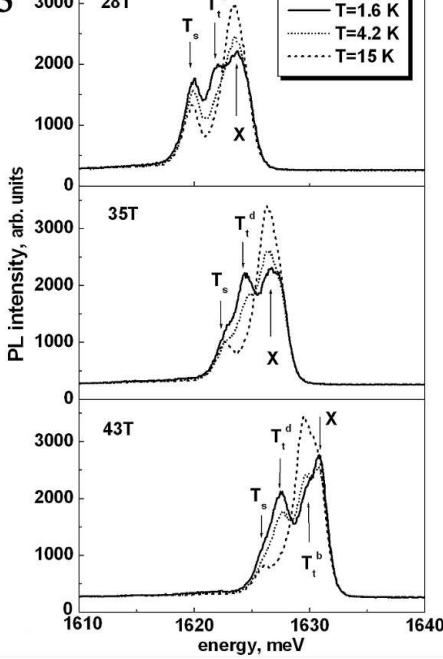

Fig. 2. Photoluminescence spectra taken from the sample with $n_{\mathrm{e}}=3 \times 10^{10} \mathrm{~cm}^{-2}$ in $\sigma^{-}$circular polarization in $5 \mathrm{~T}$ magnetic field for temperatures $1.6 \mathrm{~K}$ (solid line), $4.2 \mathrm{~K}$ (dotted line), and $15 \mathrm{~K}$ (dashed line). $T_{\mathrm{s}}$ stands for singlet trion state, $X$ - for neutral exciton.

Fig. 3. Modifications of the PL spectra with temperature at magnetic fields 28, 35, and $45 \mathrm{~T}$. Solid line $-1.6 \mathrm{~K}$, dotted line $-4.2 \mathrm{~K}$, dashed line $-15 \mathrm{~K} . X-$ the exciton line, $T_{\mathrm{s}}$ - the singlet trion line, $T_{\mathrm{t}}^{\mathrm{d}}-$ the dark triplet trion line.

polarization (Fig. 4). At $23 \mathrm{~T}$ the intensity of the $T_{\mathrm{s}}$ line at $1.6 \mathrm{~K}$ temperature is evidently higher than that of the $T_{\mathrm{s}}$ line at $4.2 \mathrm{~K}$ temperature. This corresponds to the situation when the singlet trion energy level is the ground state of the system. When the temperature rises, the ground level of the system becomes less populated and its PL decreases. But following the growth of the magnetic field one can notice that the difference between the intensities of the $T_{\mathrm{s}}$ line at 1.6 and $4.2 \mathrm{~K}$ becomes smaller. At $26-27 \mathrm{~T}$ the intensities are almost equal at these two different temperatures. If there is no difference of the intensity of the PL signal of the singlet trion state between the spectra at different temperatures, there is no difference in the population of the singlet trion level at $1.6 \mathrm{~K}$ and $4.2 \mathrm{~K}$. This means that at this range of the magnetic fields the singlet trion state is energetically very close to the next energy level, namely the triplet trion level despite the fact that their observable positions in the PL spectra are very much distinguished (about $3 \mathrm{meV})$.

To clarify this we should consider how the spectral position of the singlet trion line in photoluminescence corresponds to the energy level of this state. After recombination of an electron and hole composing a trion an electron remains on the Zeeman sublevel (upper or lower depending on the moment of the hole). Hence 

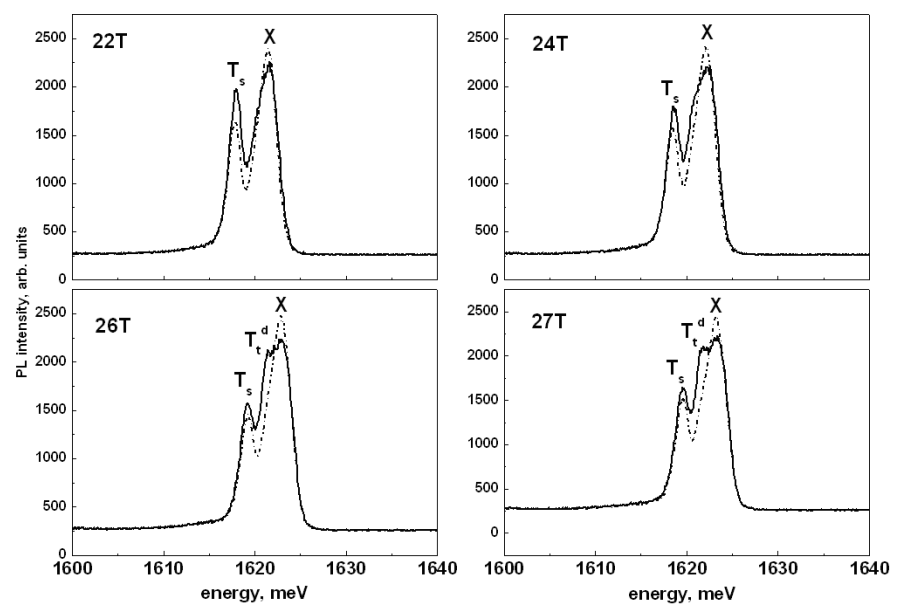

Fig. 4. PL spectra taken from the sample with $n_{\mathrm{e}}=3 \times 10^{10} \mathrm{~cm}^{-2}$ in $\sigma^{-}$polarization in $22,24,26$, and $27 \mathrm{~T}$ magnetic fields at $1.6 \mathrm{~K}$ (solid line) and $4.2 \mathrm{~K}$ (dotted line). $X$ - the exciton line, $T_{\mathrm{s}}-$ the singlet trion line, $T_{\mathrm{t}}^{\mathrm{d}}-$ the dark triplet trion line.

for the singlet trion state in $\sigma^{-}$circular polarization

$$
(\mathrm{e} \uparrow+\mathrm{e} \downarrow+\mathrm{hh} \Downarrow) \Rightarrow \mathrm{ph} \sigma^{-}+\mathrm{e} \downarrow
$$

and for the triplet trion state

$$
(\mathrm{e} \uparrow+\mathrm{e} \uparrow+\mathrm{hh} \Downarrow) \Rightarrow \mathrm{ph} \sigma^{-}+\mathrm{e} \uparrow .
$$

This leads to the conclusion that the energetic position of the trion line in the luminescence spectra differs from the real position of the trion energy level by the value of half of the electron Zeeman splitting [3]: (1/2) $g_{\mathrm{e}} \mu H$, where $g_{\mathrm{e}}$ is electron $g$-factor, $\mu$ is the Bohr magneton. Thus the total difference between the observed and the real energy positions of the singlet and triplet trion states would equal $\Delta E=g_{\mathrm{e}} \mu H$. Taking into account that the $g$-factor of the electron in CdTe is equal to -1.56 [4] one can calculate $\Delta E=2.3 \mathrm{meV}$ at $26 \mathrm{~T}$ magnetic field. This means that the real difference between the singlet and triplet energy levels is not $3 \mathrm{meV}$ as observed in experiment but only about $0.7 \mathrm{meV}$. This is confirmed by the temperature dependence of the intensity of the singlet trion PL at $1.6 \mathrm{~K}$ and $4.2 \mathrm{~K}$ temperatures.

In the fields higher than $35 \mathrm{~T}$ another PL line $T_{\mathrm{t}}^{\mathrm{b}}$, attributed to the bright triplet trion state, was observed in $\sigma^{-}$circular polarization. As the temperature grows, the $T_{\mathrm{t}}^{\mathrm{b}} \mathrm{PL}$ line becomes more intense. On the contrary, the intensity of the dark triplet trion line decreases. At the same time in magnetic fields higher than $25 \mathrm{~T}$ the intensities of the singlet trion and exciton lines depend on the temperature only slightly (Fig. 3). Thus the temperature redistribution occurs mainly between dark and bright triplet states. Since this is unusual we performed a calculation of the energy level system of the excitons and trions in order to explain this. 


\subsection{Model calculation of the exciton-trion system}

Twelve kinetic equations were used in a model calculation of the multilevel exciton-trion system. We considered four levels of hot excitons, four levels of cold excitons, two levels of singlet trion states $( \pm 3 / 2)$, two levels of triplet trion states - dark and bright $(-1 / 2)$ and the electron levels

$$
\frac{\partial n_{i}}{\partial t}=\sum_{j}\left(n_{j} w_{j i}-n_{i} w_{i j}\right)+g_{i}-\frac{n_{i}}{\tau_{i}^{\mathrm{rec}}} .
$$

Here $n_{i}$ is the exciton concentration on the $i$-th level, $w_{i j}$ is the transition rate from the $i$-th level to the $j$-th level, $g_{i}$ and $n_{i} / \tau_{i}^{\text {rec }}$ are the generation and recombination rates for the $i$-th level, correspondingly. $w_{i j}$ and $w_{j i}$ are related as: $w_{i j} / w_{j i}=$ $\exp \left(\Delta_{i j} / K T\right)$, where $\Delta_{i j}=E_{i}-E_{j}$ is the energy difference between $i$-th and $j$-th levels. The transition rate between levels $i$ and $j$ is proportional to the inverse relaxation time from the $i$-th to $j$-th level $1 / \tau_{i j}$. The electron levels are populated according to the Fermi distribution among all electron Landau levels. We also took into account the fact that the relaxation process from exciton to trion is a chemical reaction (see [1]).

The relaxation and radiative recombination processes were taken into account with characteristic time for each of the processes chosen as follows: the trion formation time (for triplet and singlet states) $\tau_{\text {form }}=10 \mathrm{ps}$, the electron spin relaxation time $\tau_{\mathrm{e}}=150 \mathrm{ps}$ and the hole spin relaxation time $\tau_{\mathrm{h}}=70 \mathrm{ps}$, the spin relaxation time of exciton as a whole $\tau_{\mathrm{X}}=30 \mathrm{ps}$, the relaxation time between bright triplet and dark triplet $\tau_{\mathrm{m}}=40 \mathrm{ps}$, the hot exciton thermalization time $\tau_{\mathrm{K}}=12 \mathrm{ps}$, the exciton and singlet trion radiative lifetime $\tau_{\text {rec }}^{\mathrm{X}}=40 \mathrm{ps}$ and $\tau_{\text {rec }}^{\mathrm{Ts}}=60 \mathrm{ps}$ respectively, the dark triplet trion radiative lifetime $\tau_{\text {rec }}^{T_{\mathrm{t}}^{\mathrm{b}}}=(50-100) \tau_{\text {rec }}^{\mathrm{Ts}}$ and the bright triplet radiative lifetime $\tau_{\mathrm{rec}}^{T_{\mathrm{t}}^{\mathrm{b}}}=80 \mathrm{ps}$. The splitting between optically dark and optically active excitons was taken to be $\delta_{\mathrm{X}}=0.2 \mathrm{meV}$ [1]. The parameters we used are similar to those in [5] for GaAs QWs and in [6] for ZnSe based QWs, only scaled according to the exciton binding energy.

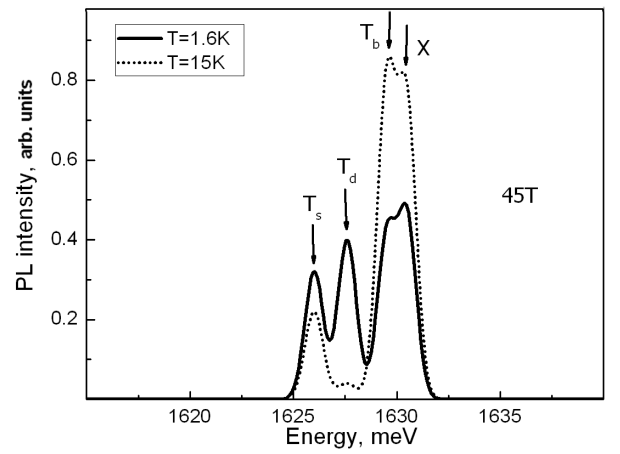

Fig. 5. Calculated PL spectra at $1.6 \mathrm{~K}$ (solid line) and $15 \mathrm{~K}$ (dotted line) in magnetic field $45 \mathrm{~T}$. 
We assumed that the relaxation time between $T_{\mathrm{t}}^{\mathrm{b}}$ and $T_{\mathrm{t}}^{\mathrm{d}}$ triplet states, being orbital momentum relaxation, is short enough (40 ps) in comparison with the electron spin relaxation time (150 ps) between triplet and singlet. Hence, the time of the temperature repopulation from $T_{\mathrm{t}}^{\mathrm{d}}$ into $T_{\mathrm{t}}^{\mathrm{b}}$ is relatively short as well. Taking into account high population of the dark triplet level $T_{\mathrm{t}}^{\mathrm{d}}$, due to preferable formation mechanism, and its long radiative recombination time (3-6 ns) it is natural to expect that temperature induced repopulation leads to the decrease in the $T_{\mathrm{t}}^{\mathrm{d}}$ line intensity, and simultaneous increase in the $T_{\mathrm{t}}^{\mathrm{b}}$ line intensity.

Using our model we have simulated the PL spectra at $1.6 \mathrm{~K}$ and $15 \mathrm{~K}$ in the magnetic field of $45 \mathrm{~T}$. Figure 5 demonstrates a qualitative agreement between calculated and experimental spectra. It is clearly seen that the intensity of dark triplet and singlet falls down with the temperature rise whereas the intensity of the bright triplet and the exciton increases.

\subsection{Reflectivity}

Optically inactive states cannot be observed in reflectivity, which means that the $T_{\mathrm{t}}^{\mathrm{d}}$ line observed in the PL should be invisible in reflectivity. At the same time the bright trion states should show up.

In Fig. 6 reflectivity spectra taken from the sample with electron concentration $n_{\mathrm{e}}=3 \times 10^{10} \mathrm{~cm}^{-2}$ are compared with the corresponding luminescence spectra in 27 and $45 \mathrm{~T}$ in $\sigma^{+}$and $\sigma^{-}$polarizations. We have found no reflectivity

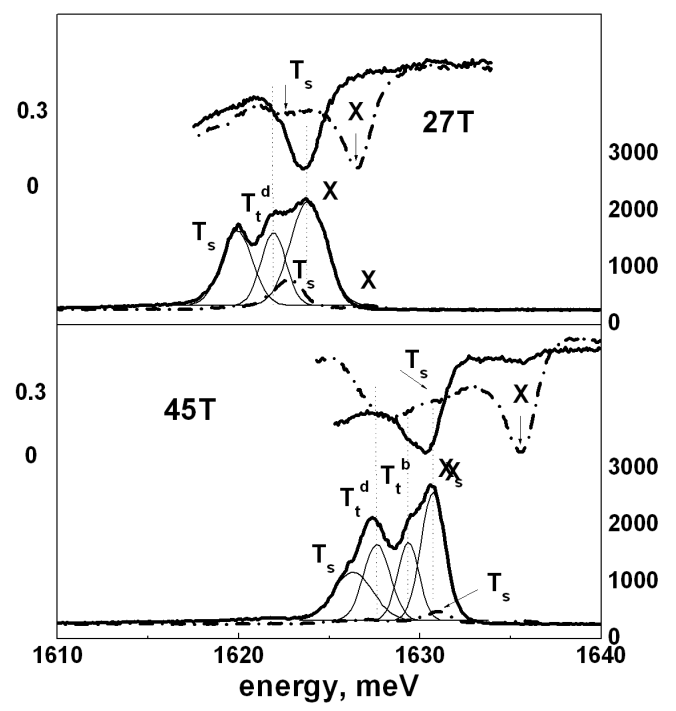

Fig. 6. Reflectivity and photoluminescence spectra taken from the sample with electron concentration $n_{\mathrm{e}}=3 \times 10^{10} \mathrm{~cm}^{-2}$ in the magnetic fields 27 and $45 \mathrm{~T}$. Heavy solid lines used for $\sigma^{-}$polarization, dashed-dotted line - for $\sigma^{+}$polarization. Light solid lines are the result of the deconvolution of the PL spectra by fitting of the Gaussian curves. Dotted straight lines are comparison of PL and reflectivity spectral features. 
line, which could correspond to the $T_{\mathrm{t}}^{\mathrm{d}}$ line in the PL spectrum. This confirms the assumption that the $T_{\mathrm{t}}^{\mathrm{d}}$ line in PL corresponds namely to the recombination of the dark triplet trion.

In high magnetic fields a shoulder marked as $T_{\mathrm{t}}^{\mathrm{b}}$ in Fig. 6 appears in the reflectivity spectra. Its energy position coincides with that of the $T_{\mathrm{t}}^{\mathrm{b}}$ line in the PL. This small shoulder corresponds to the optically active triplet trion state.

Temperature dependence of the reflectivity spectra has also been investigated. We found no strong modifications of the spectra with the temperature increase. This means that the temperature alterations only effected the kinetics of the system, which has been observed in the temperature dependent PL.

\section{Conclusions}

In the presented work we analyzed the formation mechanisms of singlet and triplet trions under the influence of high magnetic fields and considered the kinetics and thermal population of all the energy levels of the exciton-electron-trion system. This analysis allowed us to explain the behavior of the exciton and trion PL lines in CdTe-based QW at different temperatures (1.6, 4.2, and $15 \mathrm{~K}$ ) and magnetic fields. We also performed a model calculation of the exciton-trion system based on a series of kinetic equations at different temperature. The results of the calculations showed a qualitative agreement with the experimental data.

\section{References}

[1] C.R.L.P.N. Jeukens, P.C.M. Christianen, J.C. Maan, D.R. Yakovlev, W. Ossau, V.P. Kochereshko, T. Wojtowicz, G. Karczewski, J. Kossut, Phys. Rev. B 66, 235318 (2002).

[2] V. Kochereshko, D. Andronikov, A. Platonov, S.A. Crooker, T. Barrick, G. Karczewski, P. Tronc, Acta Phys. Pol. A 106, 319 (2004).

[3] K. Kheng, R.T. Cox, T. Baron, K. Seminadayar, S. Tatarenko, J. Cryst. Growth 159, 443 (1996).

[4] A.A. Sirenko, T. Ruf, M. Cardona, D.R. Yakovlev, W. Ossau, A. Waag, G. Landwehr, Phys. Rev. B 56, 2114 (1997).

[5] M.Z. Maialle, E.A. de Andrea e Silva, L.J. Sham, Phys. Rev. B 47, 15776 (1993).

[6] V.P. Kochereshko, A.V. Platonov, G.V. Mikhailov, J. Puls, F. Henneberger, D.R. Yakovlev, W. Faschinger, Int. J. Nanoscience 2, 453 (2003). 